\title{
Solvent-mediated conductance increase of dodecanethiol-stabilized gold nanoparticle monolayers
}

\author{
Patrick A. Reissner ${ }^{* 1}$, Jean-Nicolas Tisserant ${ }^{1}$, Antoni Sánchez-Ferrer ${ }^{2}$, \\ Raffaele Mezzenga ${ }^{2}$ and Andreas Stemmer ${ }^{* 1}$
}

\author{
Full Research Paper \\ Address: \\ ${ }^{1}$ ETH Zürich, Nanotechnology Group, Säumerstrasse 4, CH-8803 \\ Rüschlikon, Switzerland and ${ }^{2}$ ETH Zürich, Laboratory of Food and \\ Soft Materials, Schmelzbergstrasse 9, CH-8092 Zürich, Switzerland \\ Email: \\ Patrick A. Reissner ${ }^{*}$ - preissner@ethz.ch; Andreas Stemmer ${ }^{*}$ - \\ astemmer@ethz.ch \\ * Corresponding author \\ Keywords: \\ molecular electronics; molecular exchange; percolation networks; \\ Self-assembly
}

Beilstein J. Nanotechnol. 2016, 7, 2057-2064.

doi:10.3762/bjnano.7.196

Received: 17 August 2016

Accepted: 16 December 2016

Published: 23 December 2016

This article is part of the Thematic Series "Physics, chemistry and biology of functional nanostructures III".

Guest Editor: A. S. Sidorenko

(C) 2016 Reissner et al.; licensee Beilstein-Institut.

License and terms: see end of document.

\begin{abstract}
Gold nanoparticle monolayers provide convenient templates to study charge transport in organic molecules beyond single junction techniques. Conductance is reported to increase by several orders of magnitude following immersion of alkanethiol-stabilized gold nanoparticle monolayers in a solution containing conjugated thiol-functionalized molecules. Typically, this observation is attributed to molecular exchange. Less attention has been paid to the role of the solvent alone. Here, we report on an increase in conductance of dodecanethiol-stabilized gold nanoparticle monolayers on $\mathrm{Si} / \mathrm{SiO}_{2}$ by an average factor of 36 and 22 after immersion in pure ethanol (EtOH) and tetrahydrofuran (THF), respectively. Analysis by scanning electron microscopy (SEM) and small-angle $\mathrm{X}$-ray scattering (SAXS) reveals a solvent-induced decrease in lattice constant of close-packed monolayers. We compare the conductance of the monolayer after molecular exchange with two different oligophenylenes to shed light on the respective contribution of the solvent-induced structural change and the molecular exchange itself on the conductance increase.
\end{abstract}

\section{Introduction}

Ordered gold nanoparticle monolayers are increasingly applied as templates for molecular resistor networks [1-8]. Gold nanoparticles serve as conducting nodes and different molecules can bind to the gold nanoparticle using anchoring groups such as thiols or amines $[3,9,10]$. The conductance between neighboring nanoparticles depends on a multitude of factors including the conductivity of surrounding molecules, the type of bond between molecules and nanoparticles and the interparticle distance $[3,5,6]$. The conductivity of the entire network further depends on the percolation of charge carriers $[11,12]$. Initially, gold nanoparticles are typically stabilized by alkanethiol ligands, which are poor conductors. As reported, the conductivity of nanoparticle networks can be increased by immersing the substrate with the nanoparticle monolayer in a solution con- 
taining conjugated molecules $[3,5-7,13]$. So far, the observed overall change in conductivity has been interpreted in terms of a molecular exchange and little or no attention has been paid to the role of the solvent itself on this effect.

In the following, we demonstrate that the solvent alone can induce a structural transition responsible for a large portion of the observed increase in conductivity of micro-contact printed selfassembled gold nanoparticle monolayers.

\section{Results and Discussion}

Gold nanoparticles with an average diameter of $10.6 \mathrm{~nm}$ measured by small-angle X-ray scattering (SAXS) were synthesized [14], functionalized by 1-dodecanethiol [15], assembled to form a monolayer [16], and deposited onto an $\mathrm{Si} / \mathrm{SiO}_{2}$ substrate using a patterned poly(dimethylsiloxane) (PDMS) stamp [17]. A detailed description of all steps is provided in the methods section. The resulting pattern of the nanoparticle monolayer consists of $20 \mu \mathrm{m}$ wide lines spaced $100 \mu \mathrm{m}$ apart. An optical image of the resulting electrical devices used in this study is shown in the inset of Figure 1a. The red nanoparticle array was electrically contacted by a pair of gold electrodes separated $10 \mu \mathrm{m}$ apart.

The conductance of such devices was measured by acquiring $I-V$ curves before and after immersing them in pure solvents. All devices exhibit a linear current-voltage response before and after solvent immersion as shown in Figure 1a for a randomly picked device. The differential conductance value of each device is plotted in Figure 1b. Black data points show the conductance of the pristine devices. The average conductance of devices of batch 1 and batch 2 before immersion amounts to $2.2 \times 10^{-11} \mathrm{~S}$ and $1.4 \times 10^{-11} \mathrm{~S}$, respectively. We immersed devices of batch 1 in ethanol (EtOH) and batch 2 in tetrahydrofuran (THF) for $20 \mathrm{~h}$. Subsequent measurements of the dried devices (red data points in Figure 1) showed an average increase in conductance by a factor of 36 for $\mathrm{EtOH}$ immersion and 22 for THF immersion. Conductance changes of alkanethiol-stabilized gold nanoparticle multilayers were also reported upon exposure to solvent vapor [18,19]. However, these changes were reversible in the absence of the vapor. In our case, the change in conductance is permanent and accompanied by structural changes, as we will show in the following.

Figure 2a shows an SEM image of a pristine gold nanoparticle monolayer. Nanoparticles are hexagonally ordered within grain boundaries. Small voids between grains result from the selfassembly process. We masked voids in five SEM images at different sample locations, neglecting voids smaller than $25 \mathrm{~nm}^{2}$, as an upper limit for tolerances in interparticle distance variations. On average, these voids occupy $5 \%$ of the total area of
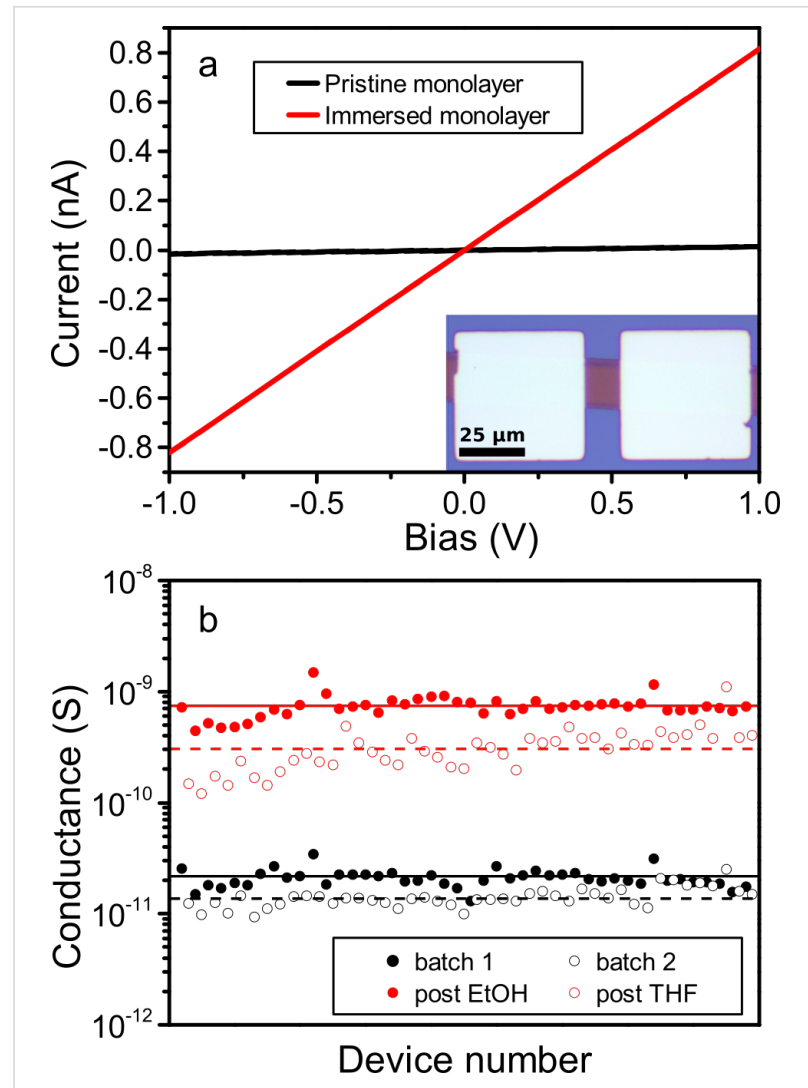

Figure 1: (a) Current-voltage relation of $20 \mu \mathrm{m} \times 10 \mu \mathrm{m}$ gold nanoparticle monolayer before (black) and after (red) immersion in $\mathrm{EtOH}$. The inset shows an optical image of a representative device. (b) Conductance values measured for 44 devices and their mean values of two different batches before (black) and after (red) solvent immersion. Full and empty symbols represent measurements of devices of batch 1 and 2 , respectively.

pristine monolayers. Larger voids evolve in nanoparticle monolayers upon immersion in EtOH or THF as demonstrated by the SEM images in Figure 2b,c. We measure an average void density of $19 \%$ and $22 \%$ after EtOH and THF immersion, respectively. For clarity, masked SEM images for void area measurements are included in the Supporting Information File 1 (Figures S1-S3). The evolution of the void distribution upon immersion is shown in Figure 2d. The average number of nanoparticles per area for the pristine monolayer, measured at five different locations, varies by $1 \%$. On average, after EtOH and THF immersion we measure $1.6 \%$ less and $2.5 \%$ more nanoparticles per unit area, respectively. The numbers of particles per area after immersion lie within one standard deviation of the measured number of particles per area on pristine monolayers. Therefore, we exclude nanoparticle removal by solvent immersion as the main reason for void formation. In combination with the measured increase in conductance these observations hint at a decreasing average particle separation upon immersion as illustrated in the schematic in Figure 2d. In Figure 3a we plot the radial power spectral density of the SEM images shown in 



Figure 2: SEM images of (a) a pristine gold nanoparticle monolayer, (b) after EtOH immersion and (c) after THF immersion. Scale bar inset is $50 \mathrm{~nm}$. (d) Histograms of the area of individual voids of the SEM images shown in (a)-(c) and a schematic illustration of solvent-induced compaction leading to void formation.

Figure 2. The extracted lattice constant for the pristine nanoparticles is $13.6 \mathrm{~nm}$ decreasing to $13.1 \mathrm{~nm}$ and $12.6 \mathrm{~nm}$ after immersion in EtOH and THF, respectively. For comparison, in an alkane chain three carbon atoms are separated by $0.25 \mathrm{~nm}$. As an estimation for the compaction of the monolayer, a reduction in lattice constant of a hexagonal unit cell by $1 \mathrm{~nm}$ would induce a $14 \%$ decrease in the occupied surface.

The results from the radial power spectral density distribution are confirmed by SAXS measurements of gold nanoparticle monolayers deposited on a pristine Kapton HN substrate, demonstrating that the observed decrease in particle separation is not linked to the substrate. Figure 3b,c show the measured and calculated intensity profiles before and after immersion in EtOH, respectively. Firstly, the form factor, $P(q)$ - blue curve in Figure $3 b, c$ - of the gold nanoparticle was obtained by fitting the intensity profile of dilute dispersions $(0.1$ and $0.01 \mathrm{wt} \%$ in water) with the polydisperse spherical nanoparticle model (Sup- porting Information File 1, Figures S4, and S5). This allowed us to evaluate the radius of the particles either from the fitting model $\left(r=5.32 \mathrm{~nm}, p=\sigma \cdot r^{-1}=0.09\right)$ or from the analysis in the Guinier regime ( $R_{\mathrm{g}}=4.46 \mathrm{~nm}$ and $R=5.76 \mathrm{~nm}$ ) (Supporting Information File 1, Figure S6). Secondly, the intensity profile for the pristine nanoparticle monolayer and for the nanoparticle monolayer immersed in EtOH was fitted - red curve in Figure $3 \mathrm{~b}, \mathrm{c}$ - by taking into account the form factor $P(q)$ of the dispersed gold nanoparticles in water - blue curve - and the structure factor $S(q)$ of the 2D version of a close-packing lattice system with paracrystalline distortion (also called lattice factor, $Z(q)$ ) - green curve [20]. Finally, from the evaluation of the first peak, $q_{1}$, from the structure factor of a $2 \mathrm{D}$ hexagonal packing, the lattice parameter, $a$, was evaluated from the relation $a=2 \pi /\left(q_{1} \cos 30^{\circ}\right)$. The lattice parameter obtained by SAXS evaluation matches the SEM measurement for the EtOH immersed sample and deviates for the pristine monolayer by $2.2 \%$. 

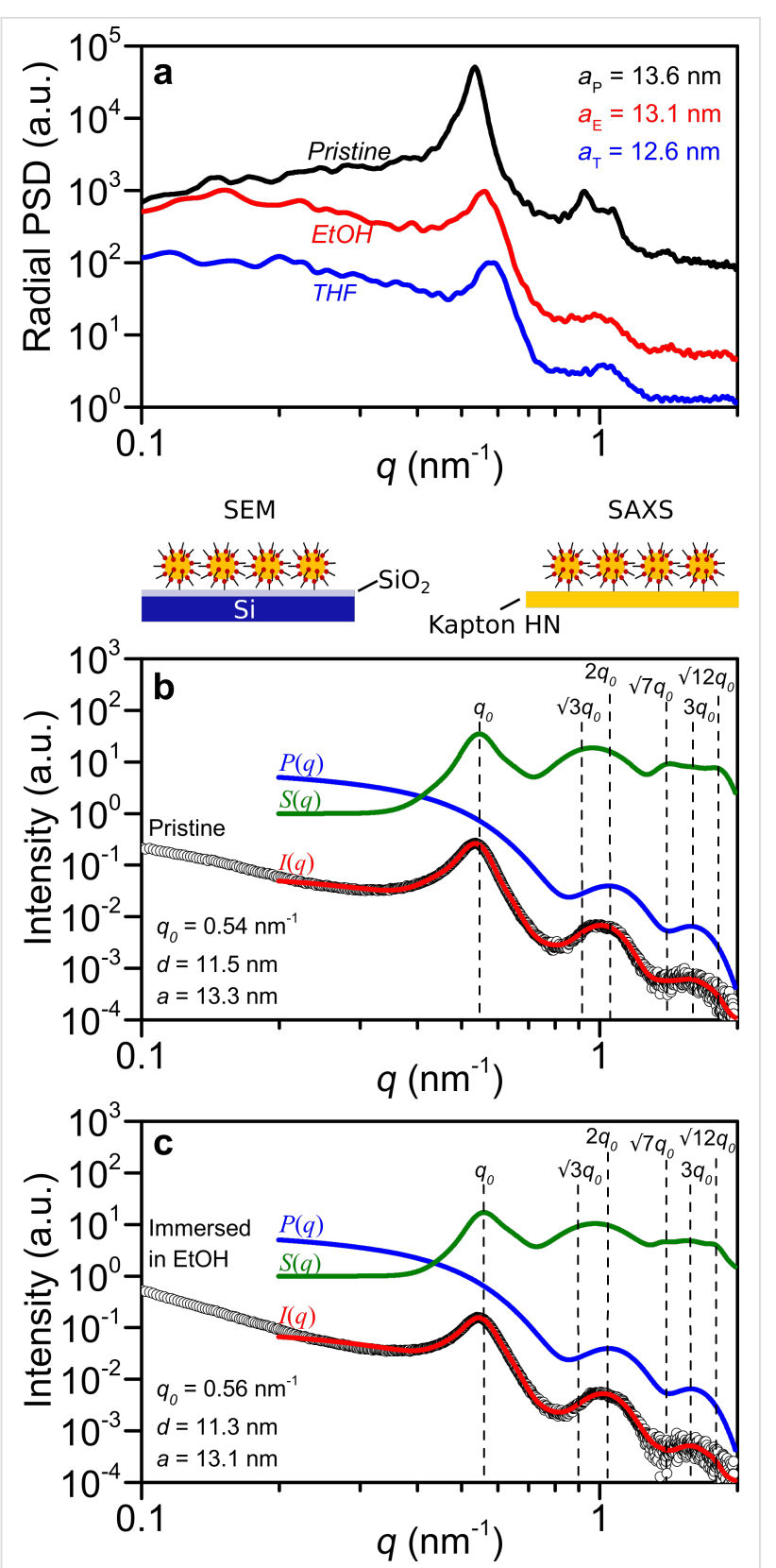

Figure 3: Evolution of gold nanoparticle monolayers upon solvent immersion measured by SEM and SAXS. (a) Radial power spectral density calculated from the respective SEM images in Figure 2 and the corresponding extracted lattice parameters. SAXS intensity profiles are shown for (b) a pristine nanoparticle monolayer and for (c) a nanoparticle monolayer immersed in EtOH. Fitting curve: $I(q)=N P(q) S(q)+$ bkg. Form factor: $P(q)=$ polydisperse spherical nanoparticle model. Structure factor: $S(q)=2 \mathrm{D}$ hexagonal packing of spheres.

A major question relates to the cause of the observed compaction and, therefore, the lateral relocation of particles on the surface. To this end, we studied the time scale on which compaction occurs. The evolution of the lattice constant as a function of immersion duration is shown for EtOH in Figure 4 for $12.3 \mathrm{~nm}$ gold nanoparticles (hydrodynamic diameter measured by dynamic light scattering).

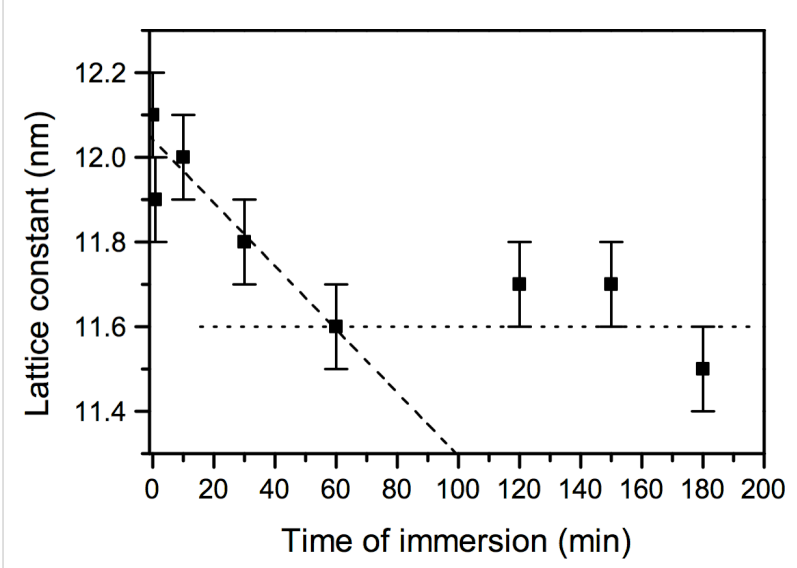

Figure 4: The change in lattice constant extracted from the radial power spectral density of SEM images taken from monolayers immersed for different durations. Dashed and dotted lines indicate trends for different durations.

We extracted the lattice constants from radial power spectral density profiles of SEM images. The lattice constant of a pristine sample coincides with the lattice constant of a monolayer immersed for $10 \mathrm{~s}$. From Figure 4, we can conclude that the compaction process is completed within one hour. This excludes that capillary forces originating from a drying solvent meniscus cause the compaction of the monolayer, because it would be independent of the immersion duration. The observed time dependence can be expected if the decrease in lattice constant is driven by an energy minimization process. Attractive van-der-Waals forces between adjacent nanoparticles can cause agglomeration of nanoparticles, which we observed after removing the alkane ligands by UV/ozone treatment. However, van-der-Waals forces rather decrease slightly in a liquid medium in comparison to air.

A slow compaction of the nanoparticle monolayer as we observed may be caused either by a collapse of alkyl tails or by partial interdigitation of alkyl tails between nanoparticles. The good solubility of 1-dodecanethiol in EtOH and THF rather points towards interdigitation than a collapse of alkyl tails. Badia et al. already suggested interdigitation of alkanes surrounding gold nanoparticles and found a temperature dependence on the alkyl chain ordering by transmission electron microscopy [21]. On flat surfaces alkane chains are known to form interdigitated layers revealed by scanning tunneling microscopy $[22,23]$. Interestingly, the measured decreases in lattice constant after solvent immersion correspond to two and four times the distance between three carbon atoms in an alkyl chain.

The conductance, $G$, of a metal-molecule-metal junction increases exponentially with decreasing interparticle distance, $d$, 
i.e., $G \propto \exp (-\beta d)$ [24-28]. This relation was shown to be valid for single junctions in alkanethiolated nanoparticle networks $[5,29]$. Dodecanethiol cannot bridge two gold nanoparticles. However, considering the gap size and the length of dodecanethiol, tails of molecules linked to opposing nanoparticles overlap. For the following calculation of conductance increase we neglect the influence of percolation of charge carriers on the overall conductance and assume a hexagonal lattice. In this case, the total conductance of the lattice is directly proportional to the conductance of a single junction [3]. The estimated conductance change originating from the measured change in lattice constant, $\Delta a$, follows then the relation $G^{\prime} / G=\exp (\beta \Delta a)$. The decay constant, $\beta$, was found to be between $1.05 \AA^{-1}$ and $0.76 \AA^{-1}$ for alkane chains with one and two chemisorbed contacts [30]. Since $\Delta a=0.5 \mathrm{~nm}$ for immersion in EtOH, the conductance would increase by a factor between 190 and 45 . On average, the conductance of our monolayers increased by 36 during EtOH immersion, which is slightly lower than the calculated values. As mentioned above, the total conductance in a nanoparticle network also depends on the number of percolation paths $[11,12]$. If the decrease in lattice constant leads to the formation of large voids and cracks in the nanoparticle monolayer, the amount of good electric connections decreases. Therefore, we measure lower conductance than estimated by just considering the decrease in lattice constant. This effect is much more pronounced for immersion in THF since more large area voids are formed as can be seen from the histograms in Figure 2d. Following the same calculation as above for THF immersion the conductance would increase by a factor ranging from 2000 to 36000 , but we only measured an average conductance increase by a factor of 22 .

In literature changes in conductance of nanoparticle monolayers after immersion in a solvent containing more conductive molecules are interpreted as an exchange of molecular ligands $[3,5,6]$. Our data showing a conductance increase by immersion in a pure solvent alone raise the question to what extent an exchange with more conductive molecules contributes to the overall increase in conductance. To this end, we immersed the samples for $20 \mathrm{~h}$ in a solvent containing molecules of higher conductivity. Dithiolated oligo(phenylenethynylene) (OPE) was dissolved in THF and $p$-terphenylthiol (TPT) was dissolved in
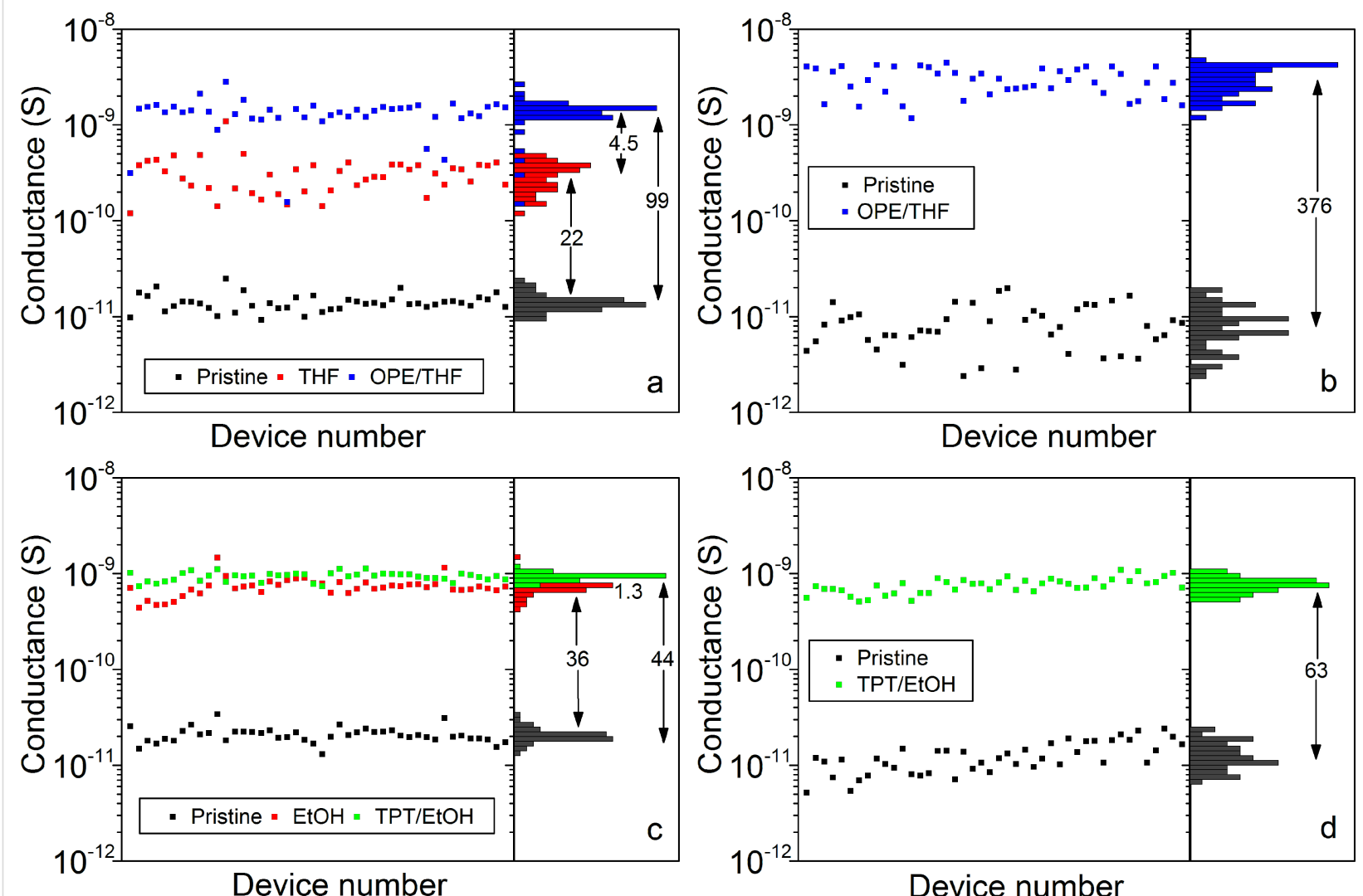

Figure 5: Influence of solvent alone and of solvent containing conjugated ligands on device conductance. (a) Pristine devices, immersed in THF, and immersed in THF with OPE. (b) Pristine devices, and immersed in THF with OPE. (c) Pristine devices, immersed in EtOH, and immersed in EtOH with TPT. (d) Pristine devices, and immersed in EtOH with TPT. Devices for each graph were fabricated on different chips. Histograms of device conductance and the relative conductance increase are shown on the right side of each plot. 
EtOH. We compare the conductance of devices which were first immersed in pure solvent (Figure 5a,c) with devices which were directly immersed in the molecule containing solvent (Figure 5b,d). The average conductance of devices that were first immersed in pure THF further increases by a factor of 4.5 after immersion in OPE/THF (Figure 5a). Pristine devices directly immersed in THF with OPE exhibit a 3.8 times higher relative conductance increase compared to sequential immersion (Figure 5b).

The measured conductance values after direct OPE/THF immersion are in agreement with previous experiments [3]. In both cases for OPE/THF immersion the conductance does not increase as much as one would expect from the decrease in lattice constant alone. Comparing the conductance values in Figure 5a,b after OPE/THF immersion with the conductance of OPE-dithiol measured by mechanical break junction experiments $\left(9.3 \times 10^{-9} \mathrm{~S}\right)$ [31], one might infer a high yield of exchanged molecules. However, the average conductance of the THF immersed devices (red data points in Figure 5a) already reaches $3 \times 10^{-10} \mathrm{~S}$, even though dodecanethiol is 100 times less conductive than OPE-dithiol [25,31].

The need to consider compaction of the nanoparticle monolayer upon solvent immersion is even more urgent in the case of TPT. We chose the TPT-monothiol to ensure that molecules do not link pairs of gold nanoparticles. Nevertheless, an increase in conductance upon exchange of alkanethiols with TPT may be expected due to $\pi-\pi$ stacking [32]. The immersion in TPT/EtOH following immersion in pure EtOH (Figure 5c) results in almost no increase in device conductance (factor 1.3). Also direct immersion of pristine devices in TPT/EtOH does not result in much higher device conductance compared to immersion in pure EtOH as shown in Figure 5d. The relative conductance increase for devices directly immersed in TPT/EtOH compared to devices first immersed in pure EtOH is 1.4 times higher. In the case of TPT/EtOH immersion, the conductance increase is dominated by the compaction of the nanoparticle monolayer upon immersion. We extracted the lattice constant $13.3 \mathrm{~nm}$ and $12.5 \mathrm{~nm}$ from the radial power spectral density (data not shown) of SEM images of the nanoparticle monolayers after TPT/EtOH and OPE/THF immersion, respectively. Therefore we exclude that molecules in the solvent inhibit compaction of the monolayer during immersion.

\section{Conclusion}

Considering all aspects we attribute a major role to the solvent in contributing to the observed conductance increase of nanoparticle monolayers undergoing liquid phase molecular exchange protocols. Solvent induced decreases in lattice constant need to be taken into account for comprehensive interpre- tation of the electronic measurements on nanoparticle monolayers. We showed here that self-assembly of nanoparticle monolayers on liquid phase followed by micro-contact printing leads to a morphology that is able to further evolve upon exposure to another solvent. This simple effect not only allows further compaction of supported nanoparticle monolayers but could equally well find application in sensing.

\section{Experimental Sample preparation}

Gold nanoparticles were synthesized in deionized water by reducing tetrachloroauric acid with trisodium citrate and tannic acid [14]. The synthesis resulted in a nanoparticle concentration of $10^{13} \mathrm{NP} / \mathrm{mL}$. The nanoparticle hydrodynamic size and polydispersity was measured by dynamic light scattering with a Malvern Zetasizer. A hydrodynamic diameter of $12.3 \mathrm{~nm}$ was measured for gold nanoparticles used for immersion duration study and $13.4 \mathrm{~nm}$ for all other experiments. $10 \mathrm{~mL}$ of the aqueous colloid were centrifuged (13000 rpm) for $30 \mathrm{~min}$ and redispersed in ethanol. $200 \mu \mathrm{L}$ 1-dodecanthiol were added to the colloidal dispersion. The nanoparticles fully precipitated after $48 \mathrm{~h}$ and the supernatant ethanol was removed with a pipette. A subsequent washing step with ethanol was performed to dispose unbound alkanethiols. The precipitated nanoparticles were dispersed in $4 \mathrm{~mL}$ of chloroform and sonicated for $10 \mathrm{~min}$ [15]. $400 \mu \mathrm{L}$ of this dispersion were deposited on a convex water surface inside a poly(tetrafluoroethylene) (PTFE) ring supported by glass slides in a petri dish [16]. After chloroform evaporation $(10 \mathrm{~min})$ the self-assembled nanoparticle monolayer was picked up by a structured PDMS (Sylgard 184, Corning) stamp with $10 \mu \mathrm{m}$ deep grooves. The $\mathrm{Si} / \mathrm{SiO}_{2}$ substrate was rinsed with acetone and isopropyl alcohol, dried with a stream of nitrogen and placed on the PDMS stamp for $10 \mathrm{~s}$ [17].

\section{Immersion}

All immersion experiments were conducted under nitrogen atmosphere for $20 \mathrm{~h}$. The solvents were bubbled for $2 \mathrm{~min}$ with nitrogen prior to immersion. For OPE/THF and TPT/EtOH immersion the molecular concentration was $1 \mathrm{mM}$. In the case of OPE, the acetyl protecting group was removed by addition of $20 \mu \mathrm{L}$ ammonia (30\%) in $3 \mathrm{~mL}$ solution while bubbling with nitrogen. The samples were immersed upside down. After immersion the samples were rinsed with THF or EtOH and dried under nitrogen flow [3].

\section{Contact deposition}

Electric contacts were applied by shadow mask evaporation. We aligned a 400 mesh TEM grid with the printed lines on the substrate and deposited $3 \mathrm{~nm}$ titanium and $65 \mathrm{~nm}$ gold by electron-beam evaporation at a pressure below $1 \times 10^{-6} \mathrm{mbar}$. 


\section{Device characterization}

We acquired $I-V$ curves in a two-probe setup on a Signatone probe station using a Keithley 6517A electrometer and a Stanford Research Systems SIM928 voltage source.

\section{SAXS measurements}

The SAXS experiments were performed using a Rigaku MicroMax-002+ microfocused beam $(40 \mathrm{~W}, 45 \mathrm{kV}, 0.88 \mathrm{~mA})$. $\mathrm{Cu} \mathrm{K} \alpha$ radiation $\left(\lambda_{\mathrm{CuK} \alpha}=1.5418 \AA\right)$ was collimated by three pinhole collimators $(0.4,0.3$, and $0.8 \mathrm{~mm})$. The scattered X-ray intensity was detected by a two-dimensional Triton-200 gas-filled X-ray detector (20 cm diameter, $200 \mu \mathrm{m}$ spatial resolution) covering a momentum transfer range of $0.1 \mathrm{~nm}^{-1}<q<2 \mathrm{~nm}^{-1}$, where $q=4 \pi \sin \theta / \lambda_{\mathrm{CuK} \alpha}$, and $2 \theta$ is the scattering angle. The scattering intensity profiles were analyzed using the software SANS \& USANS Analysis with IGOR Pro and OriginPro 10 [33]. Information about the measurement of nanoparticle size and distribution with SAXS is provided in Supporting Information File 1.

\section{Supporting Information}

\section{Supporting Information File 1}

Masking of SEM images and additional experiments.

[http://www.beilstein-journals.org/bjnano/content/

supplementary/2190-4286-7-196-S1.pdf]

\section{Acknowledgements}

The authors would like to thank Jing-Hua Tian for support in the early stage of the project and Tino Wagner for discussions. We highly appreciate assistance in the cleanroom by the staff members of the Binnig and Rohrer Nanotechnology Center.

\section{References}

1. Ancona, M.; Kruppa, W.; Rendell, R. W.; Snow, A. W.; Park, D.; Boos, J. B. Phys. Rev. B 2001, 64, No. 033408. doi:10.1103/PhysRevB.64.033408

2. Joseph, Y.; Besnard, I.; Rosenberger, M.; Guse, B.; Nothofer, H.-G.; Wessels, J. M.; Wild, U.; Knop-Gericke, A.; Su, D.; Schlögl, R.; Yasuda, A.; Vossmeyer, T. J. Phys. Chem. B 2003, 107, 7406-7413. doi:10.1021/jp030439o

3. Liao, J.; Bernard, L.; Langer, M.; Schönenberger, C.; Calame, M. Adv. Mater. 2006, 18, 2444-2447. doi:10.1002/adma.200601001

4. Blunt, M. O.; Šuvakov, M.; Pulizzi, F.; Martin, C. P.; Pauliac-Vaujour, E.; Stannard, A.; Rushforth, A. W.; Tadić, B.; Moriarty, P. Nano Lett. 2007, 7, 855-860. doi:10.1021/nl061656e

5. Dayen, J.-F.; Devid, E.; Kamalakar, M. V.; Golubev, D.; Guédon, C.; Faramarzi, V.; Doudin, B.; van der Molen, S. J. Adv. Mater. 2013, 25, 400-404. doi:10.1002/adma.201201550

6. Duan, C.; Wang, Y.; Sun, J.; Guan, C.; Grunder, S.; Mayor, M.; Peng, L.; Liao, J. Nanoscale 2013, 5, 10258-10266. doi:10.1039/c3nr02334f
7. Jafri, S. H. M.; Löfås, H.; Blom, T.; Wallner, A.; Grigoriev, A.; Ahuja, R.; Ottosson, H.; Leifer, K. Sci. Rep. 2015, 5, No. 14431. doi:10.1038/srep14431

8. Yan, Y.; Warren, S. C.; Fuller, P.; Grzybowski, B. A. Nat. Nanotechnol. 2016, 11, 603-608. doi:10.1038/nnano.2016.39

9. Nakanishi, H.; Walker, D. A.; Bishop, K. J. M.; Wesson, P. J.; Yan, Y.; Soh, S.; Swaminathan, S.; Grzybowski, B. A. Nat. Nanotechnol. 2011, 6, 740-746. doi:10.1038/nnano.2011.165

10. Dong, A.; Jiao, Y.; Milliron, D. J. ACS Nano 2013, 7, 10978-10984. doi:10.1021/nn404566b

11. Middleton, A. A.; Wingreen, N. S. Phys. Rev. Lett. 1993, 71, 3198-3201. doi:10.1103/PhysRevLett.71.3198

12. Mezzenga, R.; Ruokolainen, J.; Fredrickson, G. H.; Kramer, E. J.; Moses, D.; Heeger, A. J.; Ikkala, O. Science 2003, 299, 1872-1874. doi:10.1126/science.1081334

13. Liao, J.; Mangold, M. A.; Grunder, S.; Mayor, M.; Schönenberger, C.; Calame, M. New J. Phys. 2008, 10, No. 065019. doi:10.1088/1367-2630/10/6/065019

14. Slot, J. W.; Geuze, H. J. Eur. J. Cell Biol. 1985, 38, 87-93.

15. Huang, S.; Sakaue, H.; Shingubara, S.; Takahagi, T. Jpn. J. Appl. Phys. 1998, 37, 7198-7201. doi:10.1143/JJAP.37.7198

16. Santhanam, V.; Liu, J.; Agarwal, R.; Andres, R. P. Langmuir 2003, 19, 7881-7887. doi:10.1021/la0341761

17. Santhanam, V.; Andres, R. P. Nano Lett. 2004, 4, 41-44. doi:10.1021/nl034851r

18. Joseph, Y.; Peić, A.; Chen, X.; Michl, J.; Vossmeyer, T.; Yasuda, A. J. Phys. Chem. C 2007, 111, 12855-12859. doi:10.1021/jp072053+

19. García-Berríos, E.; Gao, T.; Woodka, M. D.; Maldonado, S.; Brunschwig, B. S.; Ellsworth, M. W.; Lewis, N. S. J. Phys. Chem. C 2010, 114, 21914-21920. doi:10.1021/jp101331g

20. Matsuoka, H.; Tanaka, H.; Hashimoto, T.; Ise, N. Phys. Rev. B 1987, 36, 1754-1765. doi:10.1103/PhysRevB.36.1754

21. Badia, A.; Singh, S.; Demers, L.; Cuccia, L.; Brown, G. R.; Lennox, R. B. Chem. - Eur. J. 1996, 2, 359-363. doi:10.1002/chem.19960020318

22. Claypool, C. L.; Faglioni, F.; Goddard, W. A., III; Gray, H. B.; Lewis, N. S.; Marcus, R. A. J. Phys. Chem. B 1997, 101, 5978-5995. doi:10.1021/jp9701799

23. Zhang, X.; Liao, L.; Wang, S.; Hu, F.; Wang, C.; Zeng, Q. Sci. Rep. 2014, 4, No. 3899. doi:10.1038/srep03899

24. Sakaguchi, H.; Hirai, A.; Iwata, F.; Sasaki, A.; Nagamura, T.; Kawata, E.; Nakabayashi, S. Appl. Phys. Lett. 2001, 79, 3708-3710. doi:10.1063/1.1421233

25. Cui, X. D.; Primak, A.; Zarate, X.; Tomfohr, J.; Sankey, O. F.; Moore, A. L.; Moore, T. A.; Gust, D.; Nagahara, L. A.; Lindsay, S. M. J. Phys. Chem. B 2002, 106, 8609-8614. doi:10.1021/jp0206065

26. Wang, W.; Lee, T.; Reed, M. A. Phys. Rev. B 2003, 68, No. 035416. doi:10.1103/PhysRevB.68.035416

27. Akkerman, H. B.; Blom, P. W. M.; de Leeuw, D. M.; de Boer, B. Nature 2006, 441, 69-72. doi:10.1038/nature04699

28. Suzuki, M.; Fujii, S.; Fujihira, M. Jpn. J. Appl. Phys. 2006, 45, 2041-2044. doi:10.1143/JJAP.45.2041

29. Zabet-Khosousi, A.; Dhirani, A.-A. Chem. Rev. 2008, 108, 4072-4124. doi:10.1021/cr0680134

30. Akkerman, H. B.; De Boer, B. J. Phys.: Condens. Matter 2008, 20 , No. 013001. doi:10.1088/0953-8984/20/01/013001

31. Huber, R.; González, M. T.; Wu, S.; Langer, M.; Grunder, S.; Horhoiu, V.; Mayor, M.; Bryce, M. R.; Wang, C.; Jitchati, R.; Schönenberger, C.; Calame, M. J. Am. Chem. Soc. 2008, 130, 1080-1084. doi:10.1021/ja0767940 
32. Wu, S.; González, M. T.; Huber, R.; Grunder, S.; Mayor, M.;

Schönenberger, C.; Calame, M. Nat. Nanotechnol. 2008, 3, 569-574.

doi:10.1038/nnano.2008.237

33. Kline, S. R. J. Appl. Crystallogr. 2006, 39, 895-900.

doi:10.1107/S0021889806035059

\section{License and Terms}

This is an Open Access article under the terms of the Creative Commons Attribution License

(http://creativecommons.org/licenses/by/4.0), which permits unrestricted use, distribution, and reproduction in any medium, provided the original work is properly cited.

The license is subject to the Beilstein Journal of

Nanotechnology terms and conditions:

(http://www.beilstein-journals.org/bjnano)

The definitive version of this article is the electronic one which can be found at:

doi:10.3762/bjnano.7.196 